\title{
Effects of TNFalpha, NOS3, MDR1 Gene Polymorphisms on Clinical Parameters, Prognosis and Survival of Multiple Myeloma Cases
}

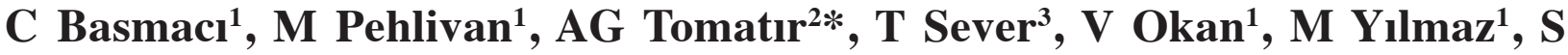 \\ Oguzkan-Balci ${ }^{3}$, S Pehlivan $^{4}$
}

\begin{abstract}
It is not clear how gene polymorphisms affecting drugs can contributes totheir efficacy in multiple myeloma (MM). We here aimed to explore associations among gene polymorphisms of tumor necrosis factor alpha (TNFalpha), nitric oxide synthesis 3 (NOS3) and multi-drug resistance 1 (MDR1), clinical parameters, prognosis and survival in MM patients treated with VAD (vincristine-adriamycine-dexamethasone), MP (mephalane-prednisolone), autolougus stem cell transplantation (ASCT), BODEC (bortezomib-dexamethasonecyclophosphamide) and TD (thalidomide-dexamethasone). We analyzed TNFalpha, NOS 3 and MDR1 in 77 patients with MM and 77 healthy controls. The genotyping was performed with PCR and/or PCR-RFLP. There was no clinically significant difference between MM and control groups when TNFalpha (-238) and (-857) and MDR1 gene polymorphisms were studied. However, the TNFalpha gene polymorphism (-308) GG genotype $(p=0.012)$ and NOS3 $(+894)$ TT genotype $(p=0.008)$ were more common in the MM group compared to healthy controls. NOS3 (VNTR) AA $(p=0.007)$ and NOS3 $(+894)$ GG genotypes $(p=0.004)$ were decreased in the MM group in contrast. In conclusion, the NOS3 $(+894)$ TT and TNFalpha $(-308)$ GG genotypes may have roles in myeloma pathogenesis.
\end{abstract}

Keywords: Multiple myeloma - NOS3 (+894, VNTR) - TNFalpha (-308, -238 and -857) - MDR1

Asian Pac J Cancer Prev, 17 (3), 1009-1014

\section{Introduction}

Genetic variation is common across the human genome. It is estimated that there are more than 7 million single nucleotide polymorphisms (SNPs) with a minor allele frequency of 5\% to $10 \%$. Although most SNPs are not functionally important, there is a subset of variants that alter the expression or function of a gene product (Banu et al., 2011).

Human tumour necrosis factor alpha $(\mathrm{TNF} \alpha)$ gene is located in the MHC locus at region 6 on chromosome 6p21.3 (http://omim.org/entry/191160). Tumor necrosis factor alpha (TNF $\alpha)$ is one of the most important cytokines in the inflammation. It effects both tissue destruction and repairment (Black et al. 1997). Not only mechanisms reducing the tendency to neoplastic transformation but also the mechanisms creativing neoplastic transformation tendency are stimulated by effect of $\mathrm{TNF} \alpha$ (Wajant et al., 2003). While some studies showed that high levels of TNF $\alpha$ suppresses the tumor angiogenesis in malign tissues, the other studies showed that TNF $\alpha$ may act as an endogenous tumor growth factor (Wajant et al., 2003). In most studies, It was shown to be a strong association between $\mathrm{TNF} \alpha$ and hematologic and non-hematologic malignancy (Black et al. 1997; Wajant et al., 2003; Olmos and Llado, 2014).

Nitric oxide (NO) is synthesized with the oxidation of L-arginin by nitric oxide synthase (NOS) (Dogra and Khullar, 2013). There are three isoforms of NOS: neuronal NOS (nNOS), endothelial NOS (eNOS) and immunological NOS (iNOS) (Nathan and Xie, 1994). eNOS on chromosome 7q 35-36 takes an important role in homeostasis (Bivalacqua et al., 2002). Therefore, variations on this gene may result in decrease of NO synthesis and cause several diseases (Heil et al., 2006; Game et al., 2013). Drug resistance is the main reason of anti-cancer treatment failure. P-glycoprotein is the main influence of multi drug resistance gene (Juliano and Ling, 1976). MDR1 phenotype is defined as over expression of MDR1 gene to produce $170 \mathrm{kDa}$ protein and p-glycoprotein (Germann, 1996). Over expression of p-glycoprotein leads to efflux of the drugs (Jamroziak et

${ }^{1}$ Department of Hematology, ${ }^{3}$ Department of Medical Biology and Genetics, Gaziantep University Faculty of Medicine, Gaziantep, ${ }^{2}$ Department of Medical Biology, Pamukkale University, Faculty of Medicine, Denizli, ${ }^{4}$ Department of Medical Biology, Istanbul University, İstanbul Medical Faculty, Istanbul,Turkey*For correspondence: tomatir@pau.edu.tr or aysegaye@hotmail.com 


\section{Basmaci et al}

al., 2005). More than 50 single nucleotide polymorphism is defined for MDR 1 gene. The most known polymorphism of MDR1 gene is MDR1 C3435T and it is a significant prognositic factor for acute lymphoblastic leukemia (Jamroziak et al., 2005).

Scientists still do not know exactly what causes most cases of multiple myeloma. However, they have made progress in understanding how certain changes in DNA can make plasma cells become cancerous (http://www. cancer.org/cancer/multiplemyeloma/). Multiple myeloma (MM) is a B-lymphoid cell disease with increased number of plasma cells in the bone marrow. It is characterized with overproduction of monoclonal immunoglobulin, osteolytic bone lesions, hypercalcemia, anemia and renal failure (Kyle, 1994; Knop, 2014). Radiation, viral agents, chronic antigenic stimulation and environmental factors are described as the etiology of disease (Ichimaru et al., 1982).

Genetic factors including single nucleotide polymorphisms (SNPs) that change cytochrome P450 (CYP) activity and epigenetic regülation that modifies CYP expression levels may contribute to the change in pharmocokinetics and adverse drug reactions (ADRs) (Nakamura et al., 2013). In this study, we investigated relationship between TNF $\alpha$, NOS3, MDR1 genes polymorphisms and clinical parameters, prognosis and survival for the VAD (Vincristine-AdriamycineDexamethasone), MP (Mephalane-Prednisolone), autologous stem cell transplantation (ASCT), BODEC (Bortezomib-Dexamethasone-Cyclophosphamide) and TD (Thalidomide-Dexamethasone) treatment of multiple myeloma.

\section{Materials and Methods}

The study was approved by the Local Ethics Committee. Of 77 patients with Multiple Myeloma, 46 of the patients enrolled to the study were male, 31 of

Table 1. Clinical Features and Treatment Regimes of Multiple Myeloma Patients

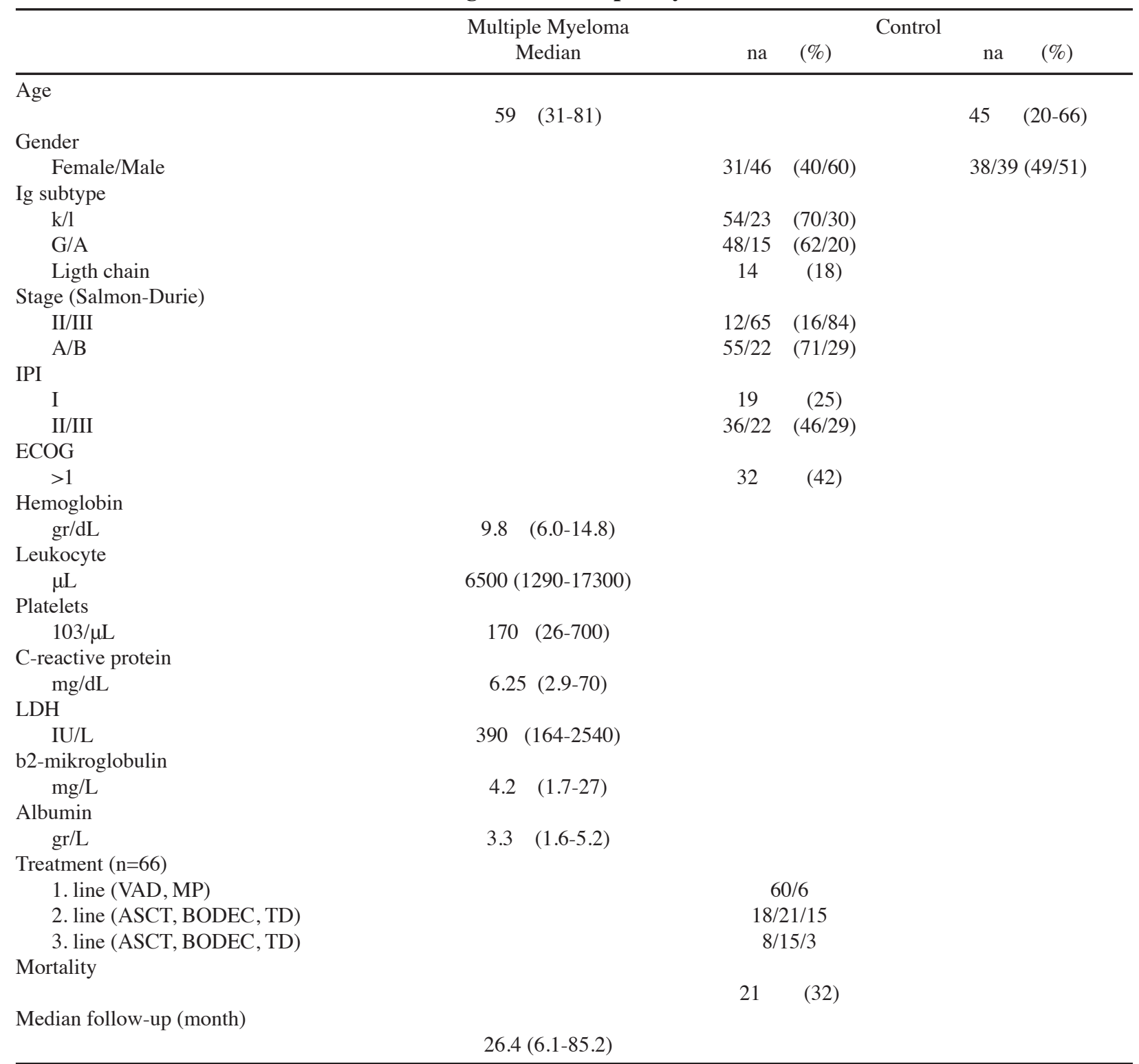

$\mathrm{n}=77$; VAD, vincristine, adriamycin ve dexamethasone; MP, melphalan, prednisolone; BODEC, bortezomib, dexamethasone, cyclophosphamide; TD, thalidomide, dexamethasone; ASCT, autologous stem cell transplantation; ECOG, performance status; LDH, lactic dehydrogenase, IPI; International prognostic index 
the patients were female. Median age was 59 (31-81 years) with the diagnosis and under treatment for MM in the Hematology Clinic, Gaziantep University Hospital. Seventy seven healthy controls were enrolled to this study, 39 of the controls were male, 38 were female and the median age was 45 (20-66 years).

\section{Polymorphism analysis}

DNA samples were isolated from peripheral blood in the healthy control group and from bone marrow in the patient group. Cytokine Genotyping was performed by polymerized chain reaction (PCR) or polymerized chain reaction restriction fragment length polymorphism (PCR-RFLP) (Akcali et al., 2010). Single nucleotide polymorphisms of the TNF $\alpha(-308,-238,-857)$, NOS3 (VNTR,-894) and MDR1 gene were analyzed by the amplifications of PCR products in gel electrophoresis.

\section{Statistical analysis}

The analyses of data's were performed by using the software SPSS (Statistical Package for the Social Sciences) for Windows (Version 13.0; SPSS, Chicago, IL). The statistical significance of differences between the patient and control groups was estimated by logistic regression analysis. Adjusted odds ratios (ORs) were calculated with logistic regression model that controlled for gender and age and were reported at $95 \%$ confidence intervals. Differences in TNF $\alpha(-308,-238,-857)$, NOS3 (-894) and MDR1 allele frequency between the control group and the patient group were compared by using the $\mathrm{X} 2$ test, and when needed, Fisher exact test was used. Survival probabilities were estimated by The KaplanMeier method and differences were compared using the log rank test (Kaplan and Meier, 1958). The Cox stepwise regression analysis was employed to confirm significance of risk factors (Cox, 1972). In multivariate analysis, we used eliminated variables stepwise (backward) by significance of less than $10 \%$. P-values $<0.05$ were considered to indicate statistical significance.

\section{Results}

Patients who were followed up for at least 6 months after the first line treatment were analyzed for response status and overall survival. Sixty of these patients received VAD (Vincristine-AdriamycineDexamethasone) treatment regime and 6 patients received MP (Mephalane-Prednisolone) treatment regime as the first line chemotherapy. Eighteen patients underwent autologous stem cell transplantation (ASCT), 21 patients received BODEC (Bortezomib-DexamethasoneCyclophosphamide) treatment regime and 15 patients received TD (Thalidomide-Dexamethasone) treatment regime as the second line therapy for treatment-resistance. Eight of the patients underwent ASCT, 15 received BODEC treatment regime and 3 received TD treatment regime for the third line therapy. The mean follow up was $26,4(6,1-85,2)$ months and mortality ratio was $32 \%$ (Table 1).

The immunoglobulins (Ig) subtypes of the patients were detected as light chain 14 (18\%), IgG 48 (62\%), IgA 15 (20\%), kappa 54 (70\%) and lambda $23(30 \%)$. According to Salmon-Durie classification 12 patients were stage II $(16 \%)$ and 65 patients $(84 \%)$ were stage III. Also 55 patients were phase A and 22 patients were phase B. According to International Staging System; 19

Table 2. Comparison of Frequencies of NOS3 (+894 ve VNTR), MDR1 and TNF A Gene Polymorphisms Between Patients with Multiple Myeloma And Healthy Controls

\begin{tabular}{|c|c|c|c|c|c|c|c|}
\hline \multicolumn{3}{|c|}{ Genotip } & \multirow{2}{*}{$\frac{\mathrm{MM}}{\mathrm{n}^{\mathrm{a}}(\%)}$} & \multirow{2}{*}{$\begin{array}{c}\text { Sagliklı } \\
\text { Kontrol } \\
\mathrm{n}^{\mathrm{a}}(\%)\end{array}$} & \multirow[t]{2}{*}{ OR } & \multirow[t]{2}{*}{$\% 95 \mathrm{CI}$} & \multirow[t]{2}{*}{$\mathrm{P}$} \\
\hline & & & & & & & \\
\hline \multirow{4}{*}{ NOS3 (VNTR) } & & AA & $40(51.9)$ & $57(74.0)$ & $0.075^{*}$ & $0.011-0.499 *$ & $0.007 *$ \\
\hline & & $\mathrm{AB}$ & $28(36.4)$ & $18(23.4)$ & $0.167 *$ & $0.024-1.165^{*}$ & $0.071 *$ \\
\hline & & BB & $9(11.7)$ & $2(2.6)$ & $0.201^{\&}$ & $0.042-0.965^{\&}$ & $0.056^{\&}$ \\
\hline & & GG & $37(48.1)$ & $51(66.2)$ & $0.041^{*}$ & $0.005-0.365^{*}$ & $0.004 *$ \\
\hline \multirow[t]{3}{*}{ NOS3 (+894) } & & GT & $25(32.5)$ & $25(32.5)$ & $1.000^{\&}$ & $0.509-1.963^{\&}$ & $1.000^{\&}$ \\
\hline & & TT & $15(19.4)$ & $1(1.3)$ & $0.049 *$ & $0.005-0.450 *$ & $0.008^{*}$ \\
\hline & & $\mathrm{CC}$ & $19(24.7)$ & $15(19.5)$ & $1.585^{*}$ & $0.621-4.047 *$ & $0.335^{*}$ \\
\hline \multirow[t]{3}{*}{ MDR1 } & & $\mathrm{CT}$ & $31(40.3)$ & $25(32.5)$ & $1.359 *$ & $0.607-3.043^{*}$ & $0.456^{*}$ \\
\hline & & $\mathrm{TT}$ & $27(35.0)$ & $37(48.0)$ & $1.713 *$ & $0.897-3.272 *$ & $0.141 *$ \\
\hline & & & 0 & 0 & & & \\
\hline \multirow[t]{3}{*}{$\mathrm{TNF} \alpha(-308)$} & AG & $18(23.4)$ & $35(45.6)$ & $0.368^{*}$ & $0.169-0.803 *$ & $0.012 *$ & \\
\hline & GG & $59(76.6)$ & $42(54.4)$ & & & & \\
\hline & $\mathrm{AA}$ & 0 & 0 & & & & \\
\hline \multirow[t]{3}{*}{$\mathrm{TNF} \alpha(-238)$} & AG & $13(16.9)$ & $10(13.0)$ & $0.883^{*}$ & $0.311-2.507 *$ & $0.815^{*}$ & \\
\hline & GG & $64(83.1)$ & $67(87.0)$ & $0.635^{\&}$ & $0.274-1.476^{\&}$ & $0.387^{\&}$ & \\
\hline & $\mathrm{TT}$ & $16(20.8)$ & $11(14.3)$ & $0.985^{*}$ & $0.349-2.778^{*}$ & $0.977 *$ & \\
\hline \multirow[t]{2}{*}{ TNF $\alpha(-857)$} & $\mathrm{TC}$ & $28(36.4)$ & $25(32.5)$ & $1.137 *$ & $0.407-3.181^{*}$ & $0.806^{*}$ & \\
\hline & $\mathrm{CC}$ & $33(42.8)$ & $41(53.2)$ & & & & \\
\hline
\end{tabular}

${ }^{\mathrm{a}} \mathrm{n}=77, *: \mathrm{OR}$; Odds ratio (\%95CI) age and sex adjusted, \&Fisher's Exact Test 
of the patients (25\%) were stage I, $36(46 \%)$ were stage II and 22 (29\%) were stage III. And according to Eastern Cooperative Oncology Group (ECOG) performance scale 32 of the patients $(42 \%)$ have higher score than 1 (Table 1). The mean hemoglobine levels, leucocyte counts, thrombocyte counts, c-reactive protein (CRP), lactate dehidrogenase (LDH), B2-microglobuline and albumine levels were 9,8 (6-14,8) gr/dl, 6500 (1290-17300) /mm3, 170x 103 (26-700x103), 6,25 (2,9-70) mg/dl, 390 (1642540) IU/L, 4,2 (1,7-27) mg/L, 3,3 (1,6-5,2) gr/L (Table 1).

TNF $\alpha$, NOS3 and MDR1 gene polymorphisms of $77 \mathrm{MM}$ patients and comparative results with the control group were summarized in Table II. In NOS3 gene VNTR polymorphism group, the AA subgroup was significantly lower in MM group in contrast to the control group $(\mathrm{p}=0,007, \mathrm{OR}=0,075)$. When we determined NOS gene $(+894)$ polymorphism results, GG genotype was significantly lower $(\mathrm{p}=0,004, \mathrm{OR}=0,041)$ and TT genotype was significantly higher $(\mathrm{p}=0,008, \mathrm{OR}=0,049)$ in $\mathrm{MM}$ group. For MDR1 gene polymorphism, there was no significant difference between patient and control groups. For TNF $\alpha$ gene (-308) polymorphism, GG genotype was statistically significant higher in MM group $(p=0,012$, $\mathrm{OR}=0,368$ ) and $\mathrm{AG}$ genotype was statistically significant lower in MM group $(\mathrm{p}=0,012, \mathrm{OR}=0,368)$. But there was no difference both (-238) and (-857) polymorphisms between control and patient groups (Table 2).

Six years overall survival was 65 months for the sixty six patients who were enrolled to the study with at least 6 months follow up and 6 years event free survival was 34,5 months, respectively. There was no significant difference between NOS3, TNF $\alpha$ and MDR1 gene polymorphism compared with the factors which have impact on overall survival such as gender, age, stage, ECOG, LDH, CRP, first line treatment and response to first line treatment. In 26 patients with Salmon-Durie stage II and in 40 patients with stage III, 6 years OS was 84 months and 56,3 months, respectively $(\mathrm{p}=0,055)$. Overall survival results in patient groups due to ISS were analyzed in 19 patients with stage I was 70 months, in 27 patients with stage II was 61 months and in 20 patients with stage III was 56,3 months $(p=0,033)$. According to ECOG; 53 patients were $\leq 1$ and their overall survival ratio was 73,2 months. Twenty three patients were ECOG $>1$ and their overall survival was 53,2 months $(\mathrm{p}=0.018)$.

\section{Discussion}

In multiple myeloma (MM), TNF $\alpha$ is involved in the generation of malignant plasma cells, as monoclonal plasma cells were produced when mononuclear cells from myeloma patients were exposed to TNF $\alpha$ and interleukin-4 in vitro (Neben et al., 2002). A study from Hungary, in $94 \mathrm{MM}$ patient and 141 control group revealed us, A allele of TNF $\alpha(-308)$ is lower expressed in MM patients. And this result can be interpreted as A allele may have a preventive effect from the disease (Kadar et al., 2008). But; in another study, TNF $\alpha(-308)$ gene polymorphism and disease severity compared between $69 \mathrm{MM}$ patient and 102 healthy control group.There was no relation between MM and this gene polymorphisms
(Iokupova et al., 2003). In our study genotypes of TNF $\alpha$ (-308) polymorphism are distributed as; AA $0(0 \%)$, AG 18 $(23,4 \%)$, GG $59(76,6 \%)$ (Table 2). The over expression of GG genotype in MM group versus control group ( $\mathrm{p}=0.012$ ) may prone to $\mathrm{MM}$ development. But there is no association between GG genotype of TNF $\alpha(-308)$ polymorphism and response to first line treatment, overall survival of ASCT-Thalidomide-Bortezomib, 6 years overall survival and 6 years event free survival.

In a study, it is reported that GG genotype of TNF $\alpha$ (-238) polymorphism may be related to early progression in $168 \mathrm{MM}$ patients formerly treated with thalidomide based regimens (Du et al., 2009). In another study, 81 refractory/relapsed MM patients treated with thalidomide based regimens were analyzed. Both TNF $\alpha$ (-308, -238) polymorphisms and TNF $\alpha$ cytokine levels were measured. The patients with G allele of TNF $\alpha(-238)$ has been found to have a shorter progression free survival. And also cytokine levels were lower before treatment $(p=0,047)$. GG allele is also found to be related with early progression but it has no effect on overall survival (Neben et al., 2002). In our study genotypes of TNF $\alpha(-238)$ are distributed as; AA $0(0 \%)$, AG $13(16,9 \%)$, GG $64(83,1 \%)$. There is no statistically significant relation between MM patient and control group. And there is no association between TNF $\alpha$ $(-238)$ polymorphism and response to first line treatment, overall survival of ASCT-Thalidomide-Bortezomib, 6 years overall survival and 6 years event free survival.

Genotypes disturbances of TNF $\alpha$ (-857) polymorphism are as; TT: 16 (20.8\%), TC: 28 (36.4\%), CC: 33 (42.8\%). No significant difference is detected between MM patients and controls for TNF $\alpha(-857)$ polymorphism. And there is no association between TNF $\alpha(-857)$ polymorphism and response to first line treatment, overall survival of ASCT-Thalidomide-Bortezomib, 6 years overall survival and 6 years event free survival.

There are several studies about relationship between NOS3 (+894, VNTR) polymorphism and several solid tumors such as breast, ovarian, colorectal, prostate and vulvary tumors. In one study, there was no relation between breast cancer and NOS3 (Ghilardi et al., 2003) but in another study, researchers suggested that NOS3 (+894) polymorphism increases nearly two-fold risk of the breast cancer without the affect of any other clinical parameter (Hefler et al., 2006). There are a few studies analyzing hematological malignancies and NOS3 $(+894)$ polymorphism in the literature. While there was no statistically significant result between non-Hodgkin lymphoma and NOS3 (+894) polymorphism (Wang et al., 2006). In our study, the distribution of NOS3 (+894) polymorphism of MM patients were detected GG genotype 37 patients $(48.1 \%)$, GT genotype $25(32,5 \%)$, TT genotype $15(19,4 \%)$ respectively (Table 2$)$. Lower expression of GG genotype $(\mathrm{p}=0,004)$ and over expression of TT genotype in MM patients $(\mathrm{p}=0,008)$ may predispose to disease. GG genotype may have a protective role on this disease. It is known that, when there is mutant $\mathrm{T}$ allele in NOS3 (+894) polymorphism, it may affect functions of the proteins that have different functions in the body (Hefler et al., 2002). In our study, TT genotype of NOS3 (+894) polymorphism is over expressed in MM group 
versus control group (19.4\% vs $1.3 \%)$. The results may attract attention an unknown role of this genotype in the pathogenesis of MM. Unfortunately, we could not find a statistically significant result between TT genotype of NOS3 (+894) polymorphism and response to first line treatment, overall survival of ASCT-ThalidomideBortezomib treatments, 6 years overall survival and 6 years event free survival.

Some studies showed that A allel of NOS3 (VNTR) gene is related with metastasis of prostate cancer and poor prognosis in patients with ovarian or vulvary cancer (Medeiros et al., 2002, Riener et al., 2004). In our study, genotypes of NOS3 (VNTR) polymorphism were distributed as; AA $40(51,9 \%), \mathrm{AB} 28(36,4 \%)$ and BB $9(11.7 \%)$. The lower expression of AA genotype in $\mathrm{MM}$ is statistically significant $(\mathrm{p}=0.007)$ and may be related to a protective effect from disease. In NOS3 (VNTR) polymorphism; $\mathrm{B}$ is the normal allele and $\mathrm{A}$ is the mutated allele. And lower expression of AA genotype of NOS3 polymorphism and changes in mRNA expression may have a role in MM pathogenesis. Still there is no association between AA genotype of NOS3 polymorphism and response to first line treatment, overall survival of ASCT-Thalidomide-Bortezomib, 6 years overall survival and 6 years event free survival.

The polymorphisms of MDR1 gene have correlation with prognosis of MM patients. The patients carrying homozygote $\mathrm{T}$ allele, heterozygote/homozygote $\mathrm{C}$ allele of C3435T position have worse prognosis (Drain et al., 2009). In an Italian study, $110 \mathrm{MM}$ patients with TT genotype of MDR1 polymorphism have better survival (Buda et al., 2007). Buda et al. (2010) said that the T/T allele carriers might be expected to show a better response to chemotherapy in the MDR1 C3435T SNP. In our study, genotypes of MDR1 polymorphism are distributed as; TT 27 (35\%), CT 31 (40.3\%), CC 19 (27.3\%). There was no significant difference between patient and control group (Table 2). And there is no association between MDR1 polymorphism and response to first line treatment, overall survival of ASCT-Thalidomide-Bortezomib, 6 years overall survival and 6 years event free survival.

When we compare clinical features and factors that affect prognosis (Age, stage (Salmon-Durie and IPI), Ig subtype, ECOG, Platelet count, LDH and CRP levels, 6-year event free survival and 6-year overall survival), the patients with IPI-3 have shorter overall survival compared to patients with IPI 1 and $2(\mathrm{p}=0.004)$ and also the patients with lower platelet count $\left(<150 \times 10^{3} / \mathrm{ml}\right)$ have worse overall survival $(\mathrm{p}=0.013)$. There is a significant relation between platelet count and overall survival (Table 1).

In conclusion, in this study, six of the MM patients who were refractory after the first line treatment, have GG genotype of TNF $\alpha$ (-238) gene polymorphism. This polymorphism may be related to poor prognosis and responsiveness to treatment. And also we found that TT genotype of NOS3 (+894) polymorphism and GG genotype of TNF $\alpha(-308)$ polymorphism are important biomarker in $\mathrm{MM}$ patients. We suggest that these polymorphisms may have a role in the pathogenesis of this disease. At the same time, our results also showed a relation between GG genotype of TNF $\alpha$ (-238) polymorphism and early progression.

\section{Acknowledgements}

This work was supported by the Research Fund of Gaziantep University (project numbers: TF.09.26).

\section{References}

Akcali A, Pehlivan S, Pehlivan M, et al (2010). TNF-alpha promoter polymorphisms in multiple sclerosis: no association with -308 and -238 alleles, but the -857 alleles in associated with the disease in Turkish patients. Int $J$ Immunogenet, 37, 91-5.

Banu C, Moise A, Arion CV, et al (2011). Cytokine Gene Polymorphisms support diagnostic monitoring of Romanian Multiple Myeloma patients. J Med Life, 4, 264-8.

Bivalacqua TJ, Champion HC, Hellstrom WJG (2002). Implications of nitric oxide synthases isoforms in the pathophysiology of Peyronie's disease. Int J Imp Res, 14, 345-2.

Black RA, Rauch CT, Kozlosky CJ, et al (1997). A metalloproteinase disintegrin that releases tumour-necrosis factor-alpha from cells. Nature, $\mathbf{3 8 5}, 729-33$.

Buda G, Maggini V, Galimberti S, et al (2007). MDR1 polymorphism influences the outcome of multiple myeloma patients. Br J Haematol. 137, 454-6.

Buda G, Ricci D, Huang CC, et al (2010). Polymorphisms in the multiple drug resistance protein 1 and in P-glycoprotein 1 are associated with time to event outcomes in patients with advanced multiple myeloma treated with bortezomib and pegylated liposomal doxorubicin. Ann Hematol. 89, 1133-40.

Cox DR (1972). Regression models and life-tables. J R Stat Soc Ser B, 34, 187-220.

Dogra S, Khullar G (2013). Tumor necrosis factor- $\alpha$ antagonists: Side effects and their management. Indian J Dermatol Venereol Leprol, 7, 35-46.

Drain S, Catherwood MA, Orr N, et al (2009). ABCB1 (MDR1) rs 1045642 is associated with increased overall survival in plasma cell myeloma. Leuk Lymphoma, 50, 566-570.

Du J, Yuan ZG, Zhang CY, et al (2009). Effect of TNF-alpha gene polymorphism on outcome of thalidomide-based regimens for multiple myeloma. Zhonghau Хие Үе Хеи Za Zhi, 30, 649-53.

Game X, Rischmann P, Arnal JF, et al (2013). Nitric oxide pathway and female lower urinary tract. Physiological and pathophysiological role]. Prog Urol, 23, 926-35.

Germann, UA (1996). P-glycoprotein--a mediator of multidrug resistance in tumour cells. Eur J Cancer. 32, 927-44.

Ghilardi G, Biondi ML, Cecchini F, et al (2003). Vasculer invasion in human breast cancer is coralated to $\mathrm{T}->786 \mathrm{C}$ polymophism of NOS3 gene. Nitric Oxide, 9, 118-22.

Hefler LA, Grimm C, Lantzsch T, et al (2006). Polymorphisms of the endothelial nitric oxide synthase gene in breast cancer. Breast Cancer Res Treat, 98, 151-55.

Hefler LA, Ludwig E, Lampe D, et al (2002). Polymorphisms of the endothelial nitric oxide synthase gene in ovarian cancer. Gynecol Oncol, 86,134-37.

Heil M, Eitenmuller I, Schmitz-Rixen T, et al (2006). Arteriogenesis versus angiogenesis: similarities and differences. J Cell Mol Med, 10, 45-55.

Ichimaru M, Ishimaru T, Mikami M, et al (1982). Multiple myeloma among atomic bomb survivors in Hiroshima and Nagasaki, 1950-76: Relationship to radiation dose absorbed by marrow. J Natl Cancer Inst, 69, 323-28.

Iokupova EV, Grinchuk OV, Kalimullina DKh, et al (2003). 


\section{Basmaci et al}

Molecular genetic analysis of the IL-6 and Tumor necrosis factor alpha gene polymorphisms in multiple myeloma. $\mathrm{Mol}$ Biol (Mosk), 37, 420-24.

Jamroziak K, Balcerczak E, Cebula B, et al (2005). Multi-drug transporter MDR1 gene polymorphism and prognosis in adult acute lymphoblastic leukemia. Pharmacol Rep, 57, 882-8.

Juliano RL, Ling VA (1976). Surface glycoprotein modulating drug permeability in Chinese hamster ovary cell mutants. Biochim Biophys Acta, 455, 152-62.

Kadar K, Kovacs M, Karadi I, et al (2008). Polymorphism of TNF-alpha and LT-alpha genes in multiple myeloma. Leuk Res. 32, 1499-504.

Kaplan EL, Meier P (1958). Nonparametric estimation from incomplete observations. J Am Stat Assoc, 53, 457-81.

Knop S (2014). The multiple myeloma - current view and perspectives. Med Monatsshr Pharm, 37, 84-92.

Kyle RA (1994). Multiple myeloma: How did it begin? Mayo Clin Proc, 69, 680-83.

Medeiros R, Morais A, Vasconcelos A, et al (2002). Endothelial nitric oxide synthase gene polymorphisms and genetic susceptibility to prostate cancer. Eur J Cancer Prev, 11, 343-50.

Nakamura K, Matsuzawa N, Ohmorı S, et al (2013). Clinical Evidence of Pharmacokinetic Changes in Thalidomide Therapy. Drug Metab Pharmacokinet, 28, 38-43.

Nathan C, Xie QW (1994). Nitric oxide synthases. Roles, tolls and controls. Cell, 78, 915-8.

Neben K, Mytilineos J, Moehler TM, et al (2002). Polymorphisms of the tumor necrosis factor- $\alpha$ gene promoter predict for outcome after thalidomide therapy in relapsed and refractory multiple myeloma. Blood, 100, 2263-65.

Olmos G, Lladó J (2014). Tumor necrosis factor alpha: a link between neuroinflammation and excitotoxicity. Mediators Inflamm, 2014, 861231 .

Riener EK, Hefler LA, Grimm C, et al (2004). Polymorphisms of the endothelial nitric oxide synthase gene in women with vulvar cancer. Gynecol Oncol, 93, 686-90.

Wajant H, Pfizenmaier K, Scheurich P (2003). Tumor necrosis factor signaling. Cell Death Differ, 10, 45-65.

Wang SS, Davis S, Cerhan JR, et al (2006). Polymorphisims in oxidative stres genes and risk for non-Hodgkin lymphoma. Carcinogenesis, 27, 1828-34. 